ISSN 2525-4804

\title{
DOSES DE FOSFÓRO NO DESENVOLVIMENTO DA CULTURA DA SOJA NA REGIÃO CENTRAL DO TOCANTINS
}

\author{
Adriano Sérgio Bernardo Queiroz ${ }^{1}$, Solange Aparecida Ságio ${ }^{2}$, Thadeu Teixeira Junior ${ }^{3}$
}

\section{Resumo:}

A produtividade de grãos de soja no Brasil é afetada pela baixa disponibilidade de fósforo no solo. Nesse sentido, o objetivo foi o de avaliar o desempenho de cultivares de soja após a aplicação de doses de fósforo. Para isso, foi instalado um experimento em casa de vegetação utilizando. o delineamento inteiramente casualizado em esquema fatorial $5 \times 2$. O primeiro fator foi constituído por cinco doses de fósforo $\left(0,50,100,200,300 \mathrm{mg} \mathrm{dm}^{-3}\right.$ de $\left.\mathrm{P}_{2} \mathrm{O}_{5}\right)$ e o segundo por duas cultivares de soja (M-SOY 9144RR e NS 8094RR). Foram avaliadas a altura de plantas e o peso da massa de grãos por planta. Os resultados foram submetidos à análise de variância pelo teste de F a 1\% de significância e teste de comparação de médias pelo teste de Scott e Knott a $1 \%$ de probabilidade. Os resultados demostraram que a variáveis altura de planta e peso de grãos, quando submetidas às doses 50, 100, 200, $300 \mathrm{mg} \mathrm{dm}^{-3} \mathrm{de} \mathrm{P}_{2} \mathrm{O}_{5}$ apresentaram resultados satisfatórios.

Palavras-chave: Adubação, Glycine max, Produtividade, Fertilidade.

\section{PHOSPHORUS DOSES IN THE DEVELOPMENT OF SOYBEAN CROP IN THE CENTRAL REGION OF TOCANTINS}

\begin{abstract}
:
The productivity of soybeans in Brazil is affected by the low availability of phosphorus in the soil. In this direction, the objective of this study was to evaluate the performance of soybean cultivars after the application of doses of phosphorus. A completely randomized design was used in a $5 \times 2$ factorial scheme for an experiment installed in a greenhouse. The first factor consisted of five doses of phosphorus $(0,50$, $100,200,300 \mathrm{mg} \mathrm{dm}^{-3}$ of $\mathrm{P}_{2} \mathrm{O}_{5}$ ) and the second for two soybean cultivars (M-SOY 9144RR and NS 8094RR). Aspects such as plant height and weight of grain mass per plant were evaluated. The results were subjected to analysis of variance by the $\mathrm{F}$ test at $1 \%$ significance and the comparison test of means by the

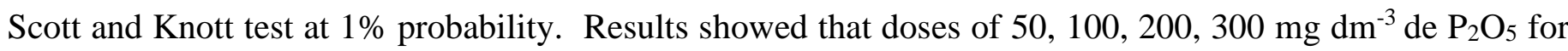
the plant height variable presented satisfactory results regardless of the variety. The same was observed for the variable grain weight per plant.
\end{abstract}

Keywords: Fertilization, Glycine max, Productivity, Fertility.

\footnotetext{
${ }^{1}$ Eng. Agrônomo, Mestre em Agroenergia - UFT; Universidade Estatual do Tocantins - UNITINS; Paraíso do Tocantins -TO; adriano.sq@unitins.br

${ }^{2}$ Eng. Agrônoma, Doutora em Fisiologia Vegetal - UFT; Universidade Federal do Tocantins; Palmas - TO; solsagio@ gmail.com

${ }^{3}$ Eng. Agrônomo, Mestre em Produção Vegetal - UniCatolica; Centro Universitário Católica do Tocantins; Palmas-TO; thadeupesquisa@mail.com
} 
O Brasil é o segundo maior produtor mundial da cultura da soja (Glycine max L. Merril), cultura que possui o maior potencial de expansão em área cultivada no Brasil, podendo, se depender das necessidades de consumo do mercado, duplicar a produção.

Com esse grande potencial, a cultura da soja tornou-se amplamente difundida em suas variadas formas de utilização e de segmentos, tendo um papel importante para a economia brasileira. Isso significa que a soja está sendo utilizada além da produção de óleo, para a produção de proteína animal e alimentação humana, consolidando a expansão da cadeia agroindustrial (Conab, 2017; Vandoir, 2017).

Nas últimas duas décadas, a soja se consolidou como a principal cultura do agronegócio brasileiro. Nesse período, o grão liderou a expansão da fronteira agrícola nacional e passou a ser cultivada em vários ambientes de produção. Com uma cadeia produtiva bem estruturada e que interage com outras, também, importantes para o agronegócio nacional, como carnes e biocombustíveis, a soja tem proporcionado o desenvolvimento socioeconômico de diversas regiões que compõem os sistemas de produção do agronegócio brasileiro (Hirakuri et al., 2017).

Para o alcance de grandes produtividades alguns fatores podem influenciar no desempenho da cultura, tais como as condições do solo e o clima; contudo, para a obtenção de boa qualidade e alta produtividade na cultura da soja, se faz necessário que as cultivares tenham um ótimo potencial genético e que os fatores ambientais sejam ideais durante todas as fases de desenvolvimento da cultura. Uma prática importante para o manejo de uma cultura é a correção e manutenção da fertilidade do solo, através da calagem e adubação (Alves, 2018).

O manejo do solo visa proporcionar condições favoráveis à semeadura e ao desenvolvimento e produção das plantas. Um elemento essencial para as plantas e que tem influência na produtividade é o fósforo. Esse elemento é de suma importância no metabolismo das plantas, pois seu papel consiste na transferência de energia da célula, na respiração e na fotossíntese. A restrição desse elemento no início do ciclo vegetativo pode resultar em limitações no desenvolvimento das plantas (Silva et al., 2018).

A exigência de fósforo pela soja varia em função do genótipo e do estádio fenológico da cultura, tendo maior demandada pela planta entre os estádios V4 e R6. A diferença na demanda de fósforo entre cultivares pode ser explicada pela diferença do seu potencial produtivo, de forma que quanto maior a produção maior tende a ser a quantidade de nutriente exportado do solo (Alves, 2018).

Solos sob vegetação de cerrados apresentam elevada acidez, alta saturação de alumínio e baixa saturação de bases. No caso do fósforo, além de se encontrar em baixas concentrações nesses solos, a sua disponibilidade para as plantas depende das reações de adsorção pelos óxidos e de precipitação com ferro e alumínio (Novais e Smyth, 1999).

Devido à sua forte interação com o solo, o fósforo é o nutriente mais utilizado na adubação de culturas do cerrado (Raij, 1991). As maiores produtividades das culturas são alcançadas quando a disponibilidade de fósforo no solo está na classe adequada ou acima dela, ou seja, com teores de fósforo de $15 \mathrm{mg} \cdot \mathrm{dm}^{-3}$ a $20 \mathrm{mg} \cdot \mathrm{dm}^{-3}$. Para que isso ocorra, são necessárias adubações corretivas com doses entre 60 a $400 \mathrm{~kg} \mathrm{ha}^{-1}$ de $\mathrm{P}_{2} \mathrm{O}_{5}$ para solos com teores muito baixos (Sousa et al., 2016).

Sabendo das exigências das culturas e dos solos de cerrado quanto ao teor de fósforo, este trabalho teve como objetivo avaliar o desempenho de características agronômicas em duas cultivares de soja, influenciadas por diferentes doses de fósforo.

O experimento foi realizado no agrícola de 2015/2016 em casa de vegetação, instalada no município de Palmas no Complexo de Ciências Agrárias (CCA) localizado na Latitude $10^{\circ} 12^{\prime} \mathrm{S}$, Longitude $48^{\circ} 21^{\prime} \mathrm{W}$ e Altitude $220 \mathrm{~m}$. 
O experimento foi instalado em vasos plásticos com capacidade de quatro litros de solo. $\mathrm{O}$ solo utilizado foi proveniente da camada arável de textura franco arenosa. Foram obtidas amostras para determinação dos dados de fertilidade por meio da realização de análises química e física (Tabela 1).

Tabela 1. Análise química e física do solo.

\begin{tabular}{|c|c|c|c|c|c|c|c|c|}
\hline $\mathbf{p H}\left(\mathbf{H}_{2} \mathrm{O}\right)$ & $\mathbf{P}$ (meh) & $\mathrm{S}-S O_{4}^{-2}$ & $\mathbf{K}^{+}$ & $\mathbf{C a}^{2+}$ & $\mathrm{Mg}^{2+}$ & $\mathbf{A l}^{3+}$ & $\mathbf{H}+\mathbf{A l}$ & M.O. \\
\hline-- & \multicolumn{2}{|c|}{--- $\mathrm{mg} \mathrm{dm}^{-3}$} & \multicolumn{5}{|c|}{ 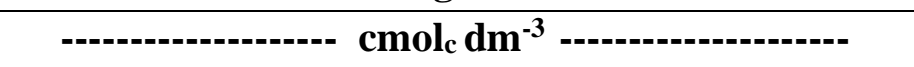 } & $\mathrm{g} \mathrm{dm}^{-3}$ \\
\hline \multirow[t]{2}{*}{5,25} & 0,62 & 8,76 & 0,03 & 0,38 & 0,23 & 0,11 & 2,62 & 11,13 \\
\hline & Areia & & \multicolumn{3}{|c|}{ Argila } & \multicolumn{3}{|c|}{ Silte } \\
\hline \multicolumn{9}{|c|}{ - } \\
\hline \multicolumn{3}{|c|}{79,3} & \multicolumn{3}{|c|}{17} & \multicolumn{3}{|c|}{3,7} \\
\hline
\end{tabular}

Após a coleta das amostras, o solo foi peneirado em malha de $4 \mathrm{~mm}$ para separação de torrões, raízes e palha. Após a colocação do solo nos vasos, foi realizada a correção da acidez. Em seguida, estes foram mantidos em incubação sob lona de plástico, por vinte dias. A adubação com potássio foi realizada segundo Novais et al., (1991).

Os tratamentos foram dispostos em esquema fatorial $5 \times 2$. Os fatores foram constituídos por cinco doses de fósforo $\left(0,50,100,200,300 \mathrm{mg} \mathrm{dm}^{-3} \mathrm{de}\right.$ $\mathrm{P}_{2} \mathrm{O}_{5}$ ) aplicados em duas cultivares de soja (M-SOY 9144RR e NS 8094RR), com cinco repetições. A dose zero correspondeu a testemunha sem a adubação fosfatada Antes da semeadura, as cultivares foram submetidas ao tratamento com fungicida (Carbendazin + Thiram $\left.{ }^{\circledR}\right)$, ultilizando-se a dosagem de $200 \mathrm{ml}$ por $100 \mathrm{~kg}$ de sementes. Posteriormente, realizou-se a inoculação do turfoso na dose de 3,0 milhões de células da bactéria Bradyrhizobium japonicum por semente, com a finalidade de obtenção de uma boa nodulação das raízes da planta e a fixação de nitrogênio pela planta. Foram semeadas dez sementes por vaso e quinze dias após a emergência foi feito o desbaste, permanecendo duas plântulas por recipiente. As plantas foram coletadas 120 dias após a emergência quando se encontravam no estádio de maturação R8, conforme escala determinada por Fehr et al. (1971). Após a coleta, foi obtida a altura de plantas (AP) com auxílio de trena graduada, medindo-se a distância da superfície do solo até a extremidade da haste principal. Posteriormente, as amostras foram trilhadas manualmente e as sementes pesadas para obtenção do peso da massa dos grãos por planta (PMG) de acordo com as metodologias das Regras de Análise de Sementes (Brasil, 2009).

Após a coleta dos dados foi realizada a análise de variância utilizando o teste F. Na detecção de efeito médio significativo entre fatores testados aplicou-se o teste Scott e Knott a $1 \%$ de significância por meio do software estatístico SISVAR (Ferreira, 2008).

Os resultados da análise de variância permitiram constatar efeitos médios significativos das doses de adubação fosfatada em relação à altura das plantas de soja. Verificou-se que ocorreu interação entre as doses de adubação e as cultivares para peso da massa dos grãos por plantas (Tabela 2). 
Tabela 2. Resumo da análise de variância das características de altura de planta (AP) e peso da massa dos grãos por plantas (PMG), duas cultivares de soja (M-SOY 9144RR e NS 8094RR) e cinco doses de adubação fosfatada $\left(\mathrm{P}_{2} \mathrm{O}_{5}\right)$.

Fonte De Variação

GL

QUADRADO MÉDIO

\begin{tabular}{cccc}
\cline { 2 - 3 } & & AP(cm) & PMG/PL(G) \\
\hline Cultivar - C & 1 & $27,38^{\mathrm{ns}}$ & $\mathbf{7 , 2 2}$ \\
Dose- D & 4 & $338,10^{*}$ & $\mathbf{7 5 , 3 5}^{*}$ \\
C X D & 4 & $106,48^{\mathrm{ns}}$ & $\mathbf{3 0 , 3 7 *}$ \\
Resíduo & 40 & 78,27 & $\mathbf{6 , 3 6}$ \\
\hline Média & - & 52,30 & $\mathbf{1 0 , 3 0}$ \\
\hline C.V. $(\%)$ & - & $\mathbf{1 6 , 9 2}$ & $\mathbf{2 4 , 4 8}$ \\
\hline
\end{tabular}

* significativo pelo teste $\mathrm{F}$ a $1 \%$ de significância.

Para o fator dose, a análise de regressão demonstrou diferença significativa, diferindo-se das demais somente a dose $0,0 \mathrm{mg} \mathrm{dm}^{-3}$ de fósforo $(42,40 \mathrm{~cm}$ ) (Figura 1). A não utilização da adubação fosfatada resultou em plantas de soja de menor porte. Por outro lado, quando se utilizou $\mathrm{P}_{2} \mathrm{O}_{5}$ como fonte de adubação fosfatada as plantas cresceram, porém, não houve diferença estatística em relação as dosagens do adubo utilizada. Apesar de não diferirem significativamente, a variedade M-SOY 9144RR demostrou a menor altura de planta para dose $0,0 \mathrm{mg} \mathrm{dm}{ }^{-3}$ de $\mathrm{P}_{2} \mathrm{O}_{5}(37,60 \mathrm{~cm})$, seguida da NS 8094RR $(47,20 \mathrm{~cm})$, únicas a apresentar média inferior a $(50 \mathrm{~cm})$, que é o mínimo exigido para o cultivo mecanizado conforme Barros et al., (2003).

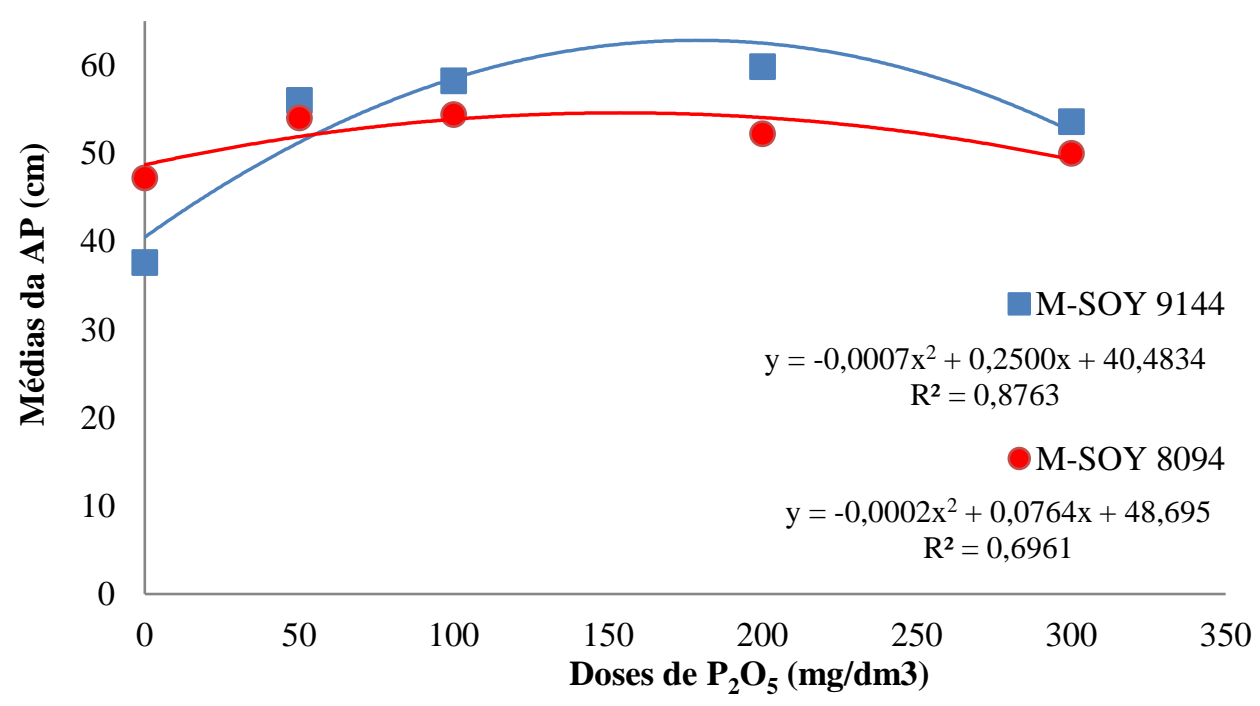

Figura 1. Médias de altura de planta $(\mathrm{AP})$ de cultivares de sojas submetidas à adubação fosfatada $\left(\mathrm{P}_{2} \mathrm{O}_{5}\right)$ em Palmas-TO, safra agrícola de 2015/2016. 
Em pesquisa realizada no estado do Goiás, Silva Júnior et al., (2017) encontraram efeito significativo nas doses de $\mathrm{P}_{2} \mathrm{O}_{5}$ na altura de plantas de soja. Já para Valadão Júnior et al., (2008), avaliando a resposta da soja em relação a doses de fósforo, verificou-se a interação significativa em relação à altura de plantas entre as cultivares estudadas e as doses de adubação fosfatada.

Cabe destacar que a altura de plantas recomendada para colheita mecanizada em solos com topografia plana é de 50 a $60 \mathrm{~cm}$ (Sediyama, 2009). Já para Rezende et al., (2005) e Garcia et al., (2007), as alturas de plantas de 50 e $120 \mathrm{~cm}$ tornamse adequada a mecanização da colheita. Assim, pode-se inferir que, com exceção da dose testemunha, as demais apresentam resultados satisfatórios independente da variedade. Por outro lado, em estudos realizados no município de Boa
Vista em Roraima, não foram encontrados efeito significativo da adubação fosfatada nas doses 0,90 , 180 e $270 \mathrm{~kg} \mathrm{ha}^{-1}$ de $\mathrm{P}_{2} \mathrm{O}_{5}$ na altura das plantas de soja (Araújo et al., 2005).

Com relação ao peso da massa dos grãos por plantas, a análise de regressão mostrou diferença significativa entre as doses aplicadas, evidenciando um incremento no rendimento de grãos de soja em função da disponibilidade de fósforo (Figura 2). Alcântara Neto et al., (2010), Valadão Júnior et al., (2008) e Guareschi et al., (2008) observaram efeito satisfatório entre doses de $\mathrm{P}_{2} \mathrm{O}_{5}$ na produtividade de grãos de soja. Araújo et al., (2005) verificaram que altas doses de fósforo, além de proporcionar maiores produtividades de grãos de soja, promove maior estímulo à nodulação. Sendo assim, maior probabilidade de fixação de nitrogênio e maior resposta ao $\mathrm{P}_{2} \mathrm{O}_{5}$.

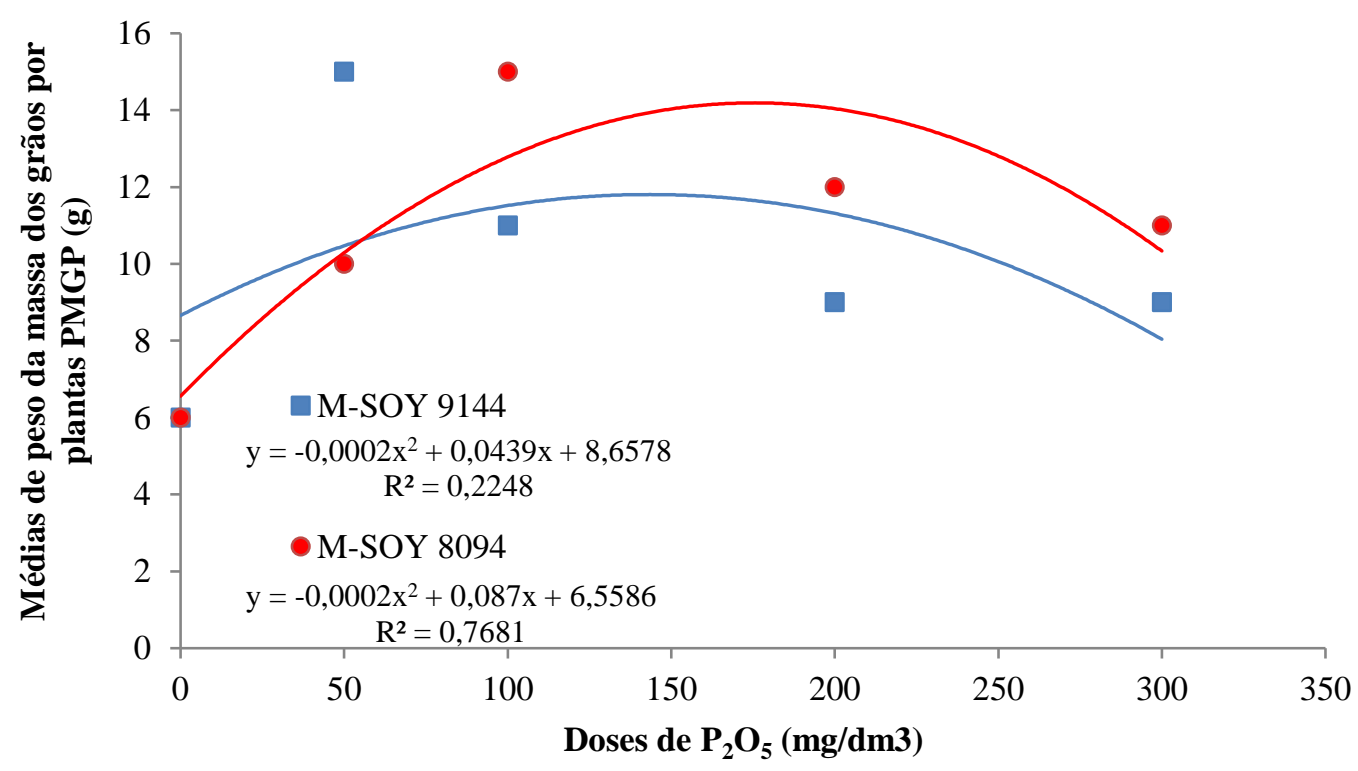

Figura 2. Médias do peso da massa dos grãos por plantas de cultivares de soja submetidas à adubação fosfatada $\left(\mathrm{P}_{2} \mathrm{O}_{5}\right)$ em Palmas-TO, safra agrícola de 2015/2016.

Com relação à média da dose $0,0 \mathrm{mg} \mathrm{dm}{ }^{-3} \mathrm{de}$ $\mathrm{P}_{2} \mathrm{O}_{5}$, verificou-se que ambas as variedades, sob baixa disponibilidade de fósforo, promoveram os menores rendimentos de grãos. A disponibilidade adequada de fósforo no solo é essencial desde os estádios iniciais de crescimento da planta. Inicialmente, as plantas vivem de suas reservas na semente, e o suprimento externo tem pouco efeito no crescimento. A falta de fósforo no início do desenvolvimento restringe o crescimento, condição 
da qual a planta não mais se recupera, limitando assim seriamente a produção. A falta de fósforo no período mais tardio do ciclo tem menor impacto na produção da cultura do que na do início (Grant et al., 2001).

Diante dos resultados obtidos podemos inferir que com a baixa disponibilidade de fósforo no solo a planta apresentará baixa estatura, o que acarretará baixo rendimento na produção. Observou-se que com o aumento das doses de fósforo no solo houve acréscimo significativo na altura de planta destacando-se para as doses 50 e 100 $\mathrm{mg} \mathrm{dm}{ }^{-3}$ de $\mathrm{P}_{2} \mathrm{O}_{5}$, que apresentaram as melhores médias em ambas as cultivares. Para a característica peso da massa dos grãos por plantas, foi observado que as melhores médias foram as doses de 50 e 100 $\mathrm{mg} \mathrm{dm}{ }^{-3}$ de $\mathrm{P}_{2} \mathrm{O}_{5}$.

\section{AGRADECIMENTOS}

Agradeço a UNITINS pela oportunidade do desenvolvimento deste trabalho.

\section{REFERÊNCIAS BIBLIOGRÁFICAS}

Alcântara Neto, F.; Amaral Gravina, G.; Souza, N.O.S.; Bezerra, A.A.C (2010). Adubação fosfatada na cultura da soja na microrregião do Alto Médio Gurguéia. Revista Ciencia Agronomica, 41(2): 266-271.

Alves, V.S.B. (2018). Eficiência de genótipos de soja no uso do fósforo (Dissertação de Mestrado). Universidade Federal de Uberlândia. Uberlândia, MG, Brasil, p. 72.

Araújo, W.F.; Arruda, R.; Dantas, R. (2005). Soybean response to phoshate fertilization. Revista Ciência Agronômica, 36:129-134.

Barros, H.B.; Peluzio, J.M.; Santos, M.M.; Brito, E.L.; Almeida, R.D. (2003). Efeito das épocas de semeadura no comportamento de cultivares de soja, no sul do estado do Tocantins. Revista Ceres 50 (291): 565-572.

BRASIL. Ministério da Agricultura, Pecuária e
Abastecimento (2009). Regras para Análise de Sementes. Brasília: Mapa/ACS. 395p.

Conab - Companhia Nacional de Abastecimento. (2017). A produtividade da soja: análise e perspectivas. vol 1. Brasília: Conab. 35p. (Compêndio de Estudos Conab. Disponível: https://www.conab.gov.br

Fehr, W.R.; Caviness, C.E.; Burmood, D.T.; Pennington, J.S. (1971). Stage of Development Descriptions for Soybeans, Glycine Max (L.) Merrill1. Crop Science, 11(6): 929. https://doi.org/10.2135/cropsci1971.0011183X0011 00060051x

Ferreira, D.F. (2008). Sisvar: um programa para análises e ensino de estatística. Revista Científica Symposium, 6(2): 36-41.

Garcia, A.; Pípolo, A.E.; Lopes, I.D.O.N.; Portugal, F.A.F. (2007). Instalação da lavoura de soja: época, cultivares, espaçamento e popuação de plantas. 51. Londrina: Embrapa, 12p. (Circular Técnica).

Grant, C.A.; Flaten, D.N.; Tomasiewicz, D.J.; Sheppard, S.C. (2001). A importância do fósforo no desenvolvimento inicial da planta. Informações Agronômicas, 91(19): 1-5.

Guareschi, R.F.; Gazolla, P.R.; Souchie, E.L.; Rocha, A.C. (2008). Adubação fosfatada e potássica na semeadura e a lanço antecipada na cultura da soja cultivada em solo de Cerrado. Semina: Ciencias Agrarias, 29(4): 769-774. https://doi.org/10.5433/1679-0359.2008v29n4p769

Hirakuri, M.; Conte, O.; Prando, A.M.; Rufino, C.F.G.; Vilardo, A.F.L.; Castro, C. (2017). Estratégia de transferência de tecnologia $\mathrm{e}$ comunicação para a sustentabilidade da sojicultura brasileira. 392. Londrina: Embrapa Soja, 26p. (Documentos)

Novais, R.F.; Smyth, T.J. (1999). Fósforo em solo e planta em condições tropicais. Viçosa: UFV/DPS, 399p.

Novais, R.F.; Neves, J.C.L.; Barros, N.F. (1991). Ensaio em ambiente controlado. In: Oliveira, A.J.; Garrido, W.E.; Araújo, J.D.; Lourenço, S. (Eds.). 
Métodos de pesquisa em fertilidade do solo. Brasília: Embrapa-SEA. p. 189-253.

Raij, B.V. (1991). Fertilidade do solo e adubação. São Paulo: Agronomica Ceres. 303p.

Rezende, P.M.; Gris, C.F.; Carvalho, J.G.; Gomes, L.L.; Bottino, L. (2005). Adubação foliar: I. Épocas de aplicação de fósforo na cultura da soja. Ciência e Agrotecnologia. 29(6): 1105-1111.

Scott, A.A.J.; Knott, M. (1974). A Cluster Analysis Method for Grouping Means in the Analysis of Variance. Biometrics, 30(3), 507-512. https://doi.org/10.2307/2529204

Sediyama, T. (2009). Tecnologias de produção e usos da soja. Londrina: Embrapa Soja. 314p.

Silva, J.; Mazon, A.; Machado, J.; Almeida, A.; Gonçalves, V. (2018). Desenvolvimento de plantas de soja submetidas a diferentes doses de fósforo aplicadas ao solo. Revista Da Jornada Da PósGraduação e Pesquisa - Congrega, 15(15): 1067 1076.

Silva Júnior, G.S.; Silva, R.C.D.; Faria, L.O.; Silva, C.S.; Pelá, A. (2017). Adubação foliar com fósforo na cultura da soja, em complementação à adubação de semeadura. In: Congresso de Ensino, Pesquisa e Extensão. 4. Anais.... Pirenópolis: UEG.

Sousa, G.; Nunes, R.D.S.; Rein, T.A.; Sciences, C. (2016). Manejo da Adubação Fosfatada para Culturas Anuais no Cerrado. 1 ed. Planaltina: Embrapa Cerrados. 10 p. (Circular Técnica 33)

Valadão Júnior, D.D.; Bergamin, A.C.; Venturoso, L.R.; Schlindwein, J.A.; Caron, B.O.; Schmidt, D. (2008). Adubação fosfatada na cultura da soja em Rondônia. Scientia Agraria. 9(3): 369-375.

Vandoir, M. (2017). Uso dos herbicidas préemergentes na cultura da soja . 1 ed. Nortox. 6 p. (Informativo Técnico Nortrox). 